\title{
Numerical Solution of Blasius Equation through Neural Networks Algorithm
}

\author{
Iftikhar Ahmad, Muhammad Bilal \\ Department of Mathematics, University of Gujrat, Gujrat, Pakistan \\ Email: dr.iftikhar@uog.edu.pk, bilalbhatti101@hotmail.com
}

Received 5 May 2014; revised 5 June 2014; accepted 11 June 2014

Copyright (C) 2014 by authors and Scientific Research Publishing Inc.

This work is licensed under the Creative Commons Attribution International License (CC BY). http://creativecommons.org/licenses/by/4.0/

c) (i) Open Access

\begin{abstract}
In this paper mathematical techniques have been used for the solution of Blasius differential equation. The method uses optimized artificial neural networks approximation with Sequential Quadratic Programming algorithm and hybrid AST-INP techniques. Numerical treatment of this problem reported in the literature is based on Shooting and Finite Differences Method, while our mathematical approach is very simple. Numerical testing showed that solutions obtained by using the proposed methods are better in accuracy than those reported in literature. Statistical analysis provided the convergence of the proposed model.
\end{abstract}

\section{Keywords}

Blasius Equation, Neural Networks, Log-Sigmoid Function, Boundary Value Problems

\section{Introduction}

Blasius differential equation is the mother of all boundary layer equations in fluid mechanics. It is almost a hundred-year-old differential equation and still an active topic among the all time researchers. Blasius derived the famous Blasius equation by using transform technique. Equation was discussed in many articles by analytical and numerical ways. Many scientists investigated interesting results of Blasius equation either from mathematical point of view or from engineering prospective. Howart numerically solved this differential equation and com- pared the results with [1]. Further, analytical solutions which are uniformly valid over the whole domain do not exist till 1999. Liao in his best paper gave the result by Homotopy Analysis Method (HPM) [2] [3]. G.I. Shishkin [4] showed asymptotic behavior of differential and difference solutions to get different scheme with a finite number of nodes for enough long interval. The standard non-homogenous Blasius equation is $u^{\prime \prime \prime}(x)+1 / 2 u^{\prime \prime}(x)=0$; with the initial and boundary condition $u(0)=0, u^{\prime}(0)=\alpha$ and $u(\infty)=1$, where $u(x)$ is dimension less stream function and is the similarity ordinate. 
The Blasius equation describes the velocity profile of fluid in a boundary layer. It is a basic equation in the fluid mechanics which appears in the study of flow of an incompressible viscous fluid over a semi-infinite plane. Blasius equation is basically derived from classical Navier Stock equation [5]-[10]. This well-known equation is investigated by many researchers to find its solution. There are many analytical and numerical methods, like Decomposition Method (DM), Iteration Method (IM), Homotopy Analysis Method (HAM) and Parameter Iteration Method (PIM), applied for solutions of differential equations.

We use Sequential Quadratic Programming (SQP) and Active set-Interior point technique (AST-INP) which is hybrid technique as optimization tools in MATLAB to solve the Blasius differential equation. In order to increase the accuracy, we repeated this proposed algorithm for several time for random selection of variable values with a moderate number of weights or variables. Moreover, a detailed statistical analysis was provided in both cases for the validity of this proposed method. In this paper we presented the numerical analysis of said equation on the bases of experiments and showed that the present solutions are highly accurate as compared to other methods.

The rest of the paper is organized as follows. In Section 2, importance of artificial neural networks and application is presented. In Section 3, we formulate the Blasius problem and propose a mathematical model for the numerical treatment of it with the help of activation function called Log-Sigmoid basis on logarithmic function. Numerical results and their graphical details are presented in next section. A brief statistical analysis and summery is presented in last section.

\section{Artificial Neural Networks}

Artificial intelligence technique is suitable in order to solve different types of differential equation. Lee and kang work with parallel processor computers to solve ordering differential equation by using Hopfield neural network model. Meads and Fernandez used B1 splines and feed forward neural network architecture to solve nonlinear and linear ordinary differential equation. Hybrid artificial neural network-nelder-mead method used by malek and shekari to solve higher order linear differential equation. A new bilaterally approach was introduced to find upper and lower bound of blasius equation by Lee. But bilaterally approach could not satisfy the boundary condition. But present study introduced the method to solve blasius differential equation which satisfies the boundary condition.

Computational models of biological brain are the example of neural networks. As the brain works, neural networks comprises large number of interconnected neurons. Each neuron has the ability to perform simple computation. As compared to biological neuron, an artificial neuron is much simpler. The construction of Artificial neural networks (ANN) is hidden in one or more layers where the factual processing is performed through weighted connections. Each neurons in the covered layer have connections to all neurons in uncovered (output) layer as shown in Figure 1. Application of such system is wide range. An artificial neural networks can learn to perform complex tasks like system identification, function approximation, trend prediction, pattern recognition and process control. Neurons in the input region only behave as buffer for dividing the input signals to neurons in the covered region.

\section{Mathematical Formulation}

The Blasius equation is given by

$$
\frac{\mathrm{d}^{3} u}{\mathrm{~d} x^{3}}+u(x) \frac{\mathrm{d}^{2} u}{\mathrm{~d} x^{2}}=0 ; \quad 0<x<\infty
$$

with boundary conditions

$$
\begin{gathered}
u(0)=\frac{\mathrm{d} u(0)}{\mathrm{d} x}=0 \\
\lim _{x \rightarrow \infty} \frac{\mathrm{d} u}{\mathrm{~d} x}=1
\end{gathered}
$$

Further Equation (1) satisfies the asymptotic condition 


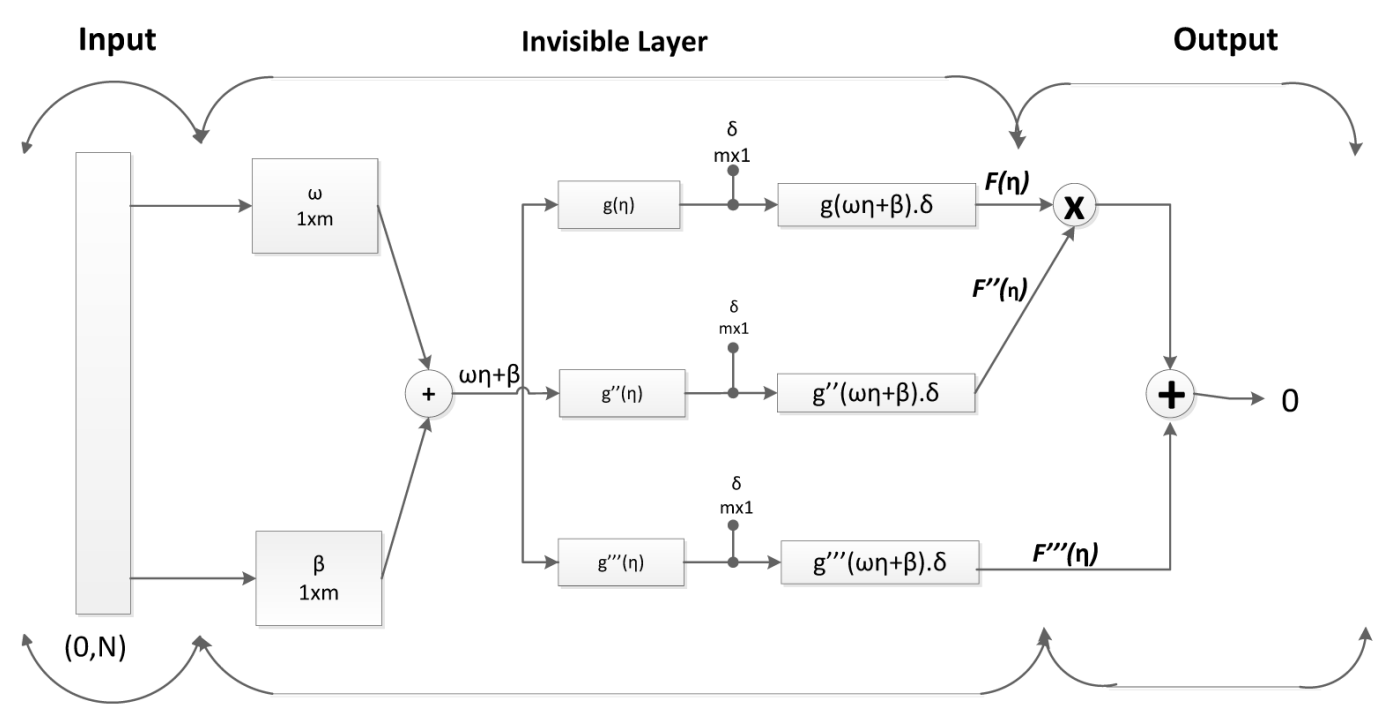

Figure 1. Neural network architecture for Blasius equation.

$$
\frac{\mathrm{d}^{2} u}{\mathrm{~d} x^{2}} \rightarrow 0 \quad \text { as } x \rightarrow \infty
$$

Put $\eta=x / x_{\infty}$ in Equations (1-3), we get

$$
\begin{gathered}
\frac{\mathrm{d}^{3} u}{\mathrm{~d} \eta^{3}}+x_{\infty} u(\eta) \frac{\mathrm{d}^{2} u}{\mathrm{~d} \eta^{2}}=0 ; 0<\eta<1 \\
u(0)=0 \\
\frac{\mathrm{d} u}{\mathrm{~d} \eta}=0 \text { at } \eta=0 \\
\frac{\mathrm{d}^{2} u}{\mathrm{~d} \eta^{2}}=0 \text { at } \eta=1
\end{gathered}
$$

We construct a mathematical model based on active set with fitness function. The system is based on numerical computation through which we obtain optimum variables of the proposed model [11]-[13].

The solution $u(\eta)$ of the differential Equation (5) along with it's derivatives, can be approximated by the following continuous relations as in neural network methodology and the activation function Log-Sigmoid is defined as

$$
L S=\sum \frac{\delta}{1+\exp (-\beta-\omega x)}
$$

Blasius equation mathematical model with the help of above activation function was developed to approximate the solution of Equations (5-8) with $2^{\text {nd }}$ and $3^{\text {rd }}$ order derivatives. Using this methodology the solution $u(\eta)$ can be approximated by

$$
\hat{u}_{L S}(\eta)=\sum_{i=1}^{m} \frac{\delta_{i}}{1+\mathrm{e}^{\left(-\beta_{i}-\omega_{i} \eta\right)}}
$$

where $m$ is the number of neurons, $\delta, \omega$, and $\beta$ are real-valued bounded adaptive parameters or weights can be expressed an array $W$ as

$$
W=\left(\delta_{1}, \delta_{2}, \cdots, \delta_{m}, \omega_{1}, \omega_{2}, \cdots, \omega_{m}, \beta_{1}, \beta_{2}, \cdots, \beta_{m}\right)
$$

The second and third order derivatives of solution $u(\eta)$ can be approximation by the continuous relations 


$$
\begin{gathered}
\hat{u}_{L S}^{(2)}(\eta)=\sum_{i=1}^{m} \delta_{i} \omega_{i}^{2}\left[\frac{2 \mathrm{e}^{\left(-2 \beta_{i}-2 \omega_{i} \eta\right)}}{\left(1+\mathrm{e}^{\left(-\beta_{i}-\omega_{i} \eta\right)}\right)^{3}}-\frac{\mathrm{e}^{\left(-\beta_{i}-\omega_{i} \eta\right)}}{\left(1+\mathrm{e}^{\left(-\beta_{i}-\omega_{i}(\eta)\right)}\right)^{2}}\right] \\
\hat{u}_{L S}^{(3)}(\eta)=\sum_{i=1}^{m} \delta_{i} \omega_{i}^{3}\left[\frac{6 \mathrm{e}^{\left(-3 \beta_{i}-3 \omega_{i} \eta\right)}}{\left(1+\mathrm{e}^{\left(-\beta_{i}-\omega_{i} \eta\right)}\right)^{4}}-\frac{6 \mathrm{e}^{\left(-2 \beta_{i}-2 \omega_{i} \eta\right)}}{\left(1+\mathrm{e}^{\left(-\beta_{i}-\omega_{i} \eta\right)}\right)^{3}}+\frac{\mathrm{e}^{\left(-\beta_{i}-\omega_{i} \eta\right)}}{\left(1+\mathrm{e}^{\left(-\beta_{i}-\omega_{i}(\eta)\right)}\right)^{2}}\right]
\end{gathered}
$$

where $u^{(2)}$ and $u^{(3)}$ represented 2nd and 3rd derivative with respect to $\eta$ respectively.

The mathematical model for Equation (5) can be formulated by a linear combination of networks Equations $(10-12)$ is called a differential equation neural networks.

The fitness function for proposed model $\in$ has been formulated for the Equations (5-8) using Mathematical model by defining the error as the sum of mean squared errors:

$$
\epsilon=\epsilon_{1}+\epsilon_{2}+\epsilon_{3}
$$

The error term $\epsilon_{1}$ is connected with the physical problem (5) with $x_{\infty}=1$ is given as:

$$
\epsilon_{1}=\operatorname{AVERAGE}\left[\hat{u}_{i}^{(3)}+\hat{u}_{i} \hat{u}_{i}^{(2)}\right]^{2} \text {; for } i=1, N+1 .
$$

where $\hat{u}_{i}=\hat{u}\left(\eta_{i}\right)$, interval $[0,1]$ with step size $h=0.1$ is divided by $N+1$ subintervals i.e., $\left[\eta_{1}, \eta_{2}\right],\left[\eta_{2}, \eta_{3}\right], \cdots,\left[\eta_{N}, \eta_{N+1}\right]$.

And $\in_{2}$ for initial values can be defined as

$$
\epsilon_{2}=\operatorname{AVERAGE}\left(u_{0}+u_{0}^{(1)}\right)^{2}
$$

For $\eta=1$ we have $\epsilon_{3}=\left[u^{(2)}\right]^{2}$.

\section{Optimization Procedure for Numerical Solution}

Furthermore, we provide some detail about the procedural steps for the optimization in MATLAB built-in function is given below. The generic flow diagram of the overall process is shown in Figure 2.

Step 1: Initialization:

A vector with randomly generated bounded real values of length equal to the number of weights in each Mathematical model acts as the starting point for each solver:

$$
W=\left(\delta_{1}, \delta_{2}, \cdots, \delta_{m}, \omega_{1}, \omega_{2}, \cdots, \omega_{m}, \beta_{1}, \beta_{2} \cdots, \beta_{m}\right)
$$

Here $m$ represents the number of neurons.

Step 2: Fitness Evaluation:

The MATLAB built-in function for constrained optimization problems is invoked for each model.

\section{Step 3: Termination Criteria:}

Terminate the execution of the solver, if any of the following criteria is satisfied:

- required level of predefined fitness achieved, i.e., $\in \leq 10^{-14}$.

- total number of iterations executed, as listed in Table 1.

Step 4: Storage:

Save the final optimal weights (variables) along with fitness values and computational time taken by the algorithm.

Step 5: Statistical Analysis:

Repeat steps 1 to 4 for sufficiently large number of times to perform an effective and reliable statistical analysis.

\section{Numerical Results}

In this section, we show the output of the proposed method by the numerical results of Blasius equation. Further, 


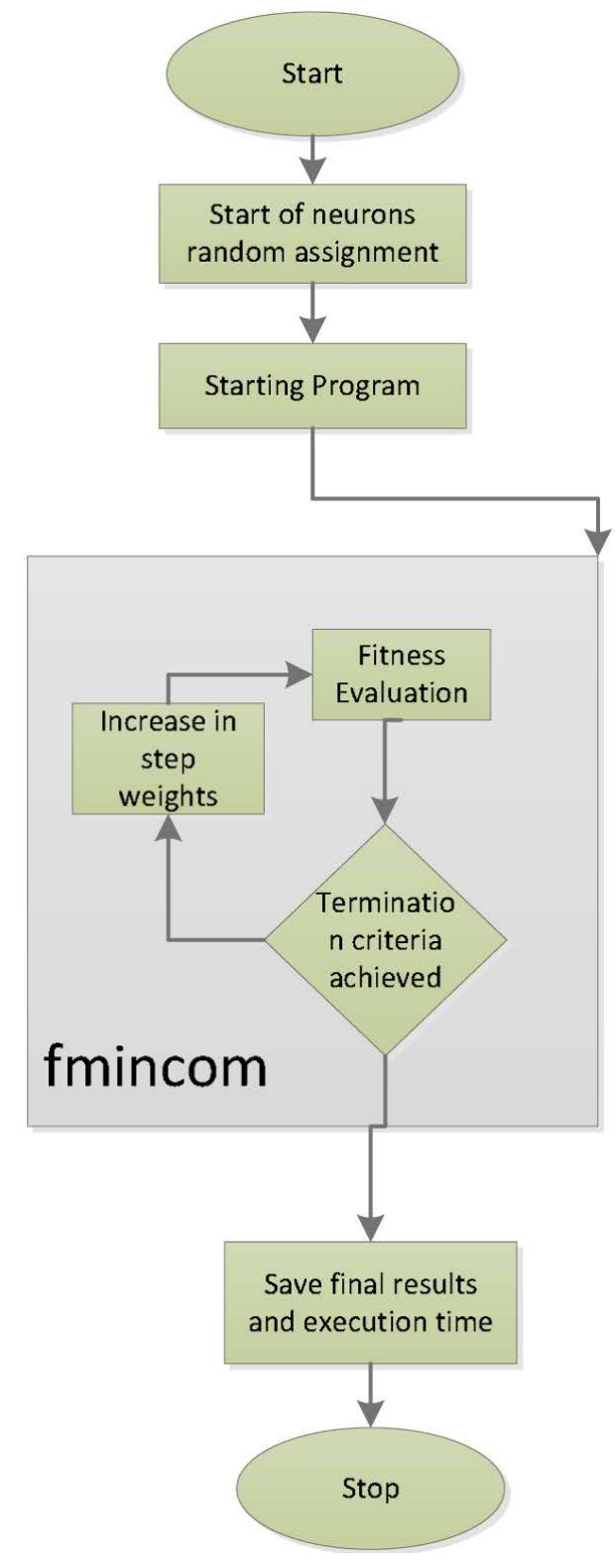

Figure 2. Flow chart of Blasius equation model.

Table 1. Parameter settings for the function "fmincon" in MATLAB simulations.

\begin{tabular}{cc}
\hline Parameters & Settings/Values \\
"Fin Diff Type" & "Central” \\
Start Point generation & Randomly between $(0,1)$ \\
Hessian & BFGS \\
Minimum Perturbation & $10^{-08}$ \\
Total Start Points & 100 \\
Max Iterations & 1700 \\
Max Fun Evals & 1000000 \\
Start Point Size & $30-60$ \\
$X$ Tolrence & $10^{-14}$ \\
Scaling & Objective and Constraints \\
\hline
\end{tabular}


we calculate the values of $u_{R F}$ called reference solution with MATHEMATICA, approximate solution $\hat{u}_{L S}$ and the absolute error function $\left|u_{R F}-\hat{u}_{L S}\right|$ with hybrid AST-INP and SQP algorithms. Furthermore, we provided the statistical analysis with several time run of optimization tools for Mean, Median, STD and Variance tabulated in Table 2 and Table 3 for both cases. Which showed that the present solution is highly accurate as compared to others methods present in literature.

\section{Statistical Analysis and Discussion}

On the basis of the simulations and results obtained in the previous section, it can be concluded that Blasius differential equation can be solved by stochastic computational intelligence technique, like SQP optimization algorithm, AST-INP hybrid technique, supported with simulating annealing. The differential equation neural networks trained by SQP algorithm and AST-INP are better stochastic optimizers as compared to other algorithms. The statistical analysis for this case with AST-INP and SQP algorithm are tabulated in Table 2 and Table 3. These results showed the better accuracy of numerical data with reference solution. We presented minimum value, mean, median and STD for the accuracy of our solver for 300 time multi-runs with minimum time as shown in Table 2 and Table 3.

Table 2. Statistical analysis of solution of Blasius equation with hybrid (AST-INP).

\begin{tabular}{cccccc}
\hline$\eta$ & Exact & Mean & Median & STD & Var \\
0 & 0 & $-8.24 \mathrm{E}-04$ & $-5.79 \mathrm{E}-04$ & 0.0017 & $2.98 \mathrm{E}-06$ \\
0.1 & 0.005215 & 0.0046 & 0.0047 & 0.0016 & $2.63 \mathrm{E}-06$ \\
0.2 & 0.020856 & 0.0204 & 0.0205 & 0.0016 & $2.58 \mathrm{E}-06$ \\
0.3 & 0.046911 & 0.0466 & 0.0466 & 0.0016 & $2.72 \mathrm{E}-06$ \\
0.4 & 0.083345 & 0.0832 & 0.0831 & 0.0017 & $2.95 \mathrm{E}-06$ \\
0.5 & 0.130089 & 0.13 & 0.1299 & 0.0018 & $3.21 \mathrm{E}-06$ \\
0.6 & 0.187034 & 0.1869 & 0.1868 & 0.0019 & $3.42 \mathrm{E}-06$ \\
0.7 & 0.254021 & 0.2539 & 0.2538 & 0.0019 & $3.57 \mathrm{E}-06$ \\
0.8 & 0.330829 & 0.3307 & 0.3306 & 0.0019 & $3.63 \mathrm{E}-06$ \\
0.9 & 0.417178 & 0.417 & 0.417 & 0.0019 & $3.61 \mathrm{E}-06$ \\
\hline
\end{tabular}

Table 3. Statistical analysis of solution of Blasius equation with SQP.

\begin{tabular}{|c|c|c|c|c|c|c|}
\hline$\eta$ & Min & Max & Mean & Median & STD & Var \\
\hline 0 & -0.00546 & 0.00454 & -0.00064 & -0.00045 & 0.0013 & $1.66 \mathrm{E}-06$ \\
\hline 0.1 & $-9.03 \mathrm{E}-06$ & $9.99 \mathrm{E}-03$ & $4.68 \mathrm{E}-03$ & $4.82 \mathrm{E}-03$ & 0.0013 & $1.65 \mathrm{E}-06$ \\
\hline 0.2 & 0.01509 & 0.02587 & 0.0204 & 0.02055 & 0.0013 & $1.70 \mathrm{E}-06$ \\
\hline 0.3 & 0.04059 & 0.05216 & 0.04652 & 0.04665 & 0.0013 & $1.79 \mathrm{E}-06$ \\
\hline 0.4 & 0.07648 & 0.08881 & 0.083 & 0.08315 & 0.0014 & $1.91 \mathrm{E}-06$ \\
\hline 0.5 & 0.1227 & 0.1358 & 0.1298 & 0.1299 & 0.0014 & $2.04 \mathrm{E}-06$ \\
\hline 0.6 & 0.1791 & 0.1929 & 0.1867 & 0.1869 & 0.0015 & $2.18 \mathrm{E}-06$ \\
\hline 0.7 & 0.2456 & 0.26 & 0.2537 & 0.2539 & 0.0015 & $2.32 \mathrm{E}-06$ \\
\hline 0.8 & 0.322 & 0.3369 & 0.3305 & 0.3307 & 0.0016 & $2.44 \mathrm{E}-06$ \\
\hline 0.9 & 0.4079 & 0.4233 & 0.4168 & 0.417 & 0.0016 & $2.55 \mathrm{E}-06$ \\
\hline 1 & 0.5031 & 0.5189 & 0.5122 & 0.5125 & 0.0016 & $2.64 \mathrm{E}-06$ \\
\hline
\end{tabular}


Furthermore, Figure 3 and Figure 4 represented the 2-dimensional and 3-dimensional view of weights or variables obtained from SQP optimizer. Similarly, Figure 5 and Figure 6 represented the 2-dimensional and

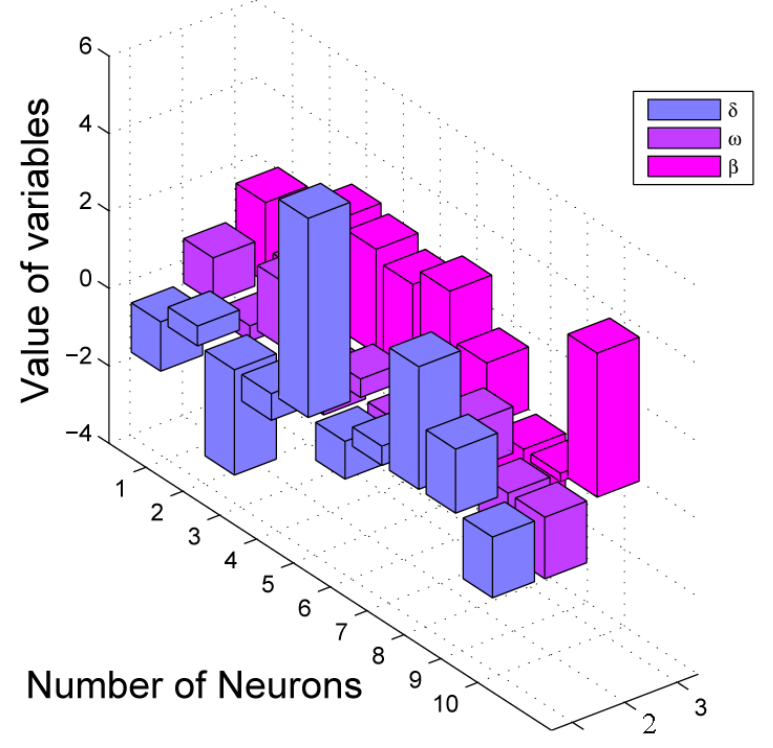

Figure 3. A 3D view of neural network model strained with SQP for Blasius equation.

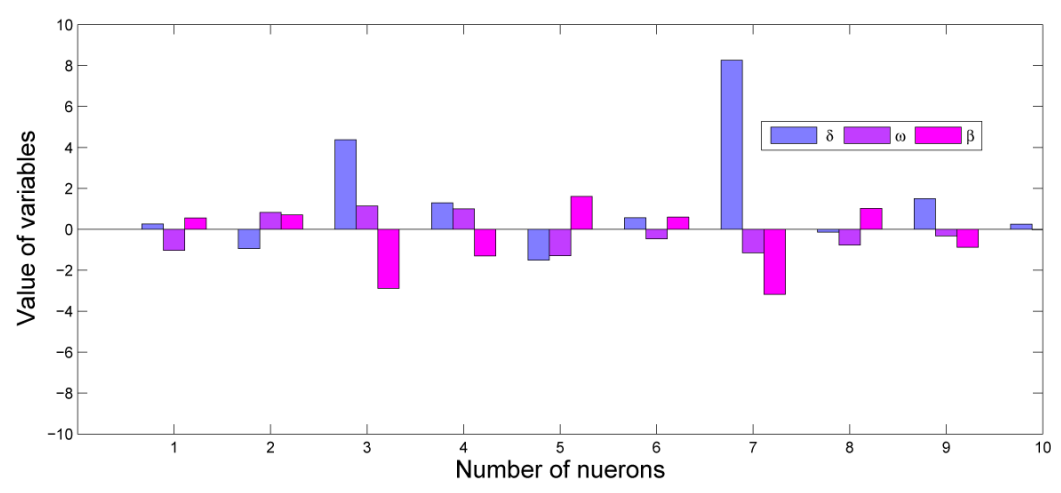

Figure 4. A 2D view of a set of variables of neural network model strained with SQP for Blasius equation.

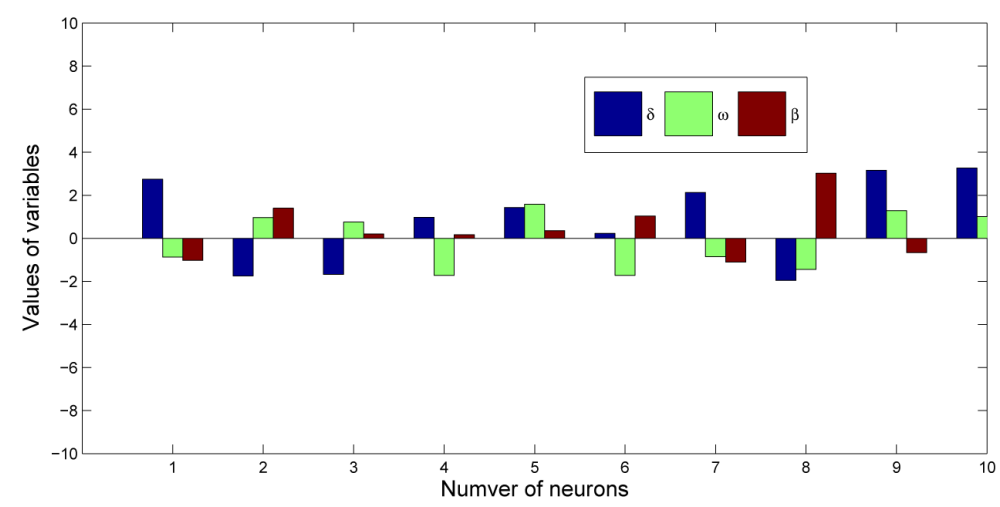

Figure 5. A 2D view of neural network model strained with hybrid (AST-INP) for Blasius equation. 
3-dimensional view of weights or variables obtained from AST-INP optimizer. In Figure 7, it is also shown that the confidence level of absolute error at $10^{-20}$ is 90 percent and maximum values lies between $10^{-20}$ and $10^{-18}$. We have shown the Chi-squares distribution fitness with normal form to the numerical data through several time simulation as shown in Figure 8. Furthermore, in Figure 9, we presented the comparison of reported results mean with exact solution (reference solution).

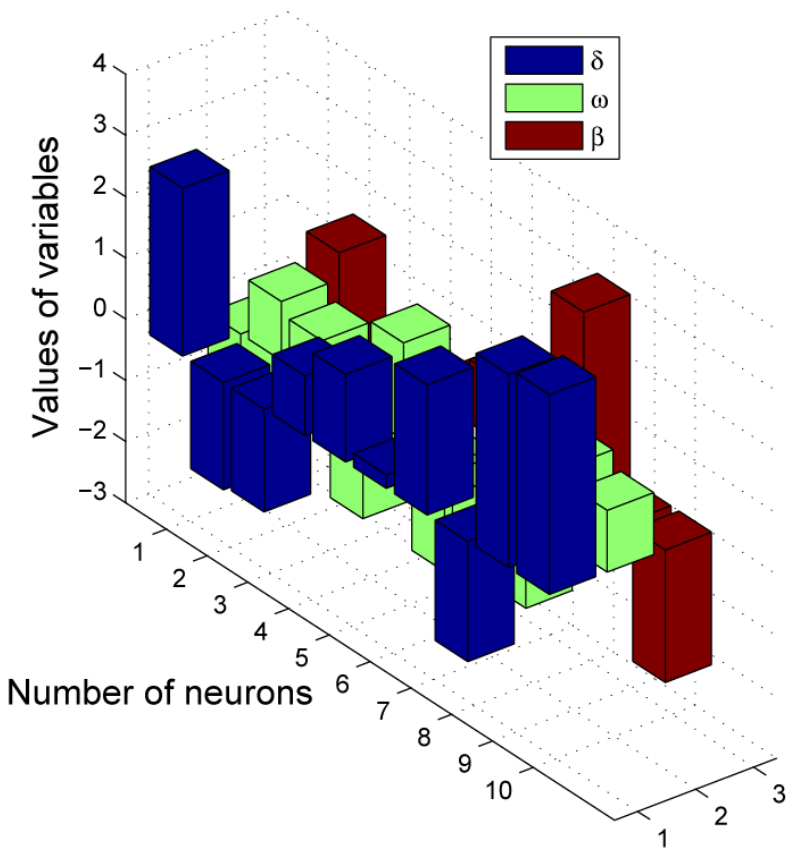

Figure 6. A $3 \mathrm{D}$ view of set of variables of neural network model strained with hybrid (AST-INP) for Blasius equation.

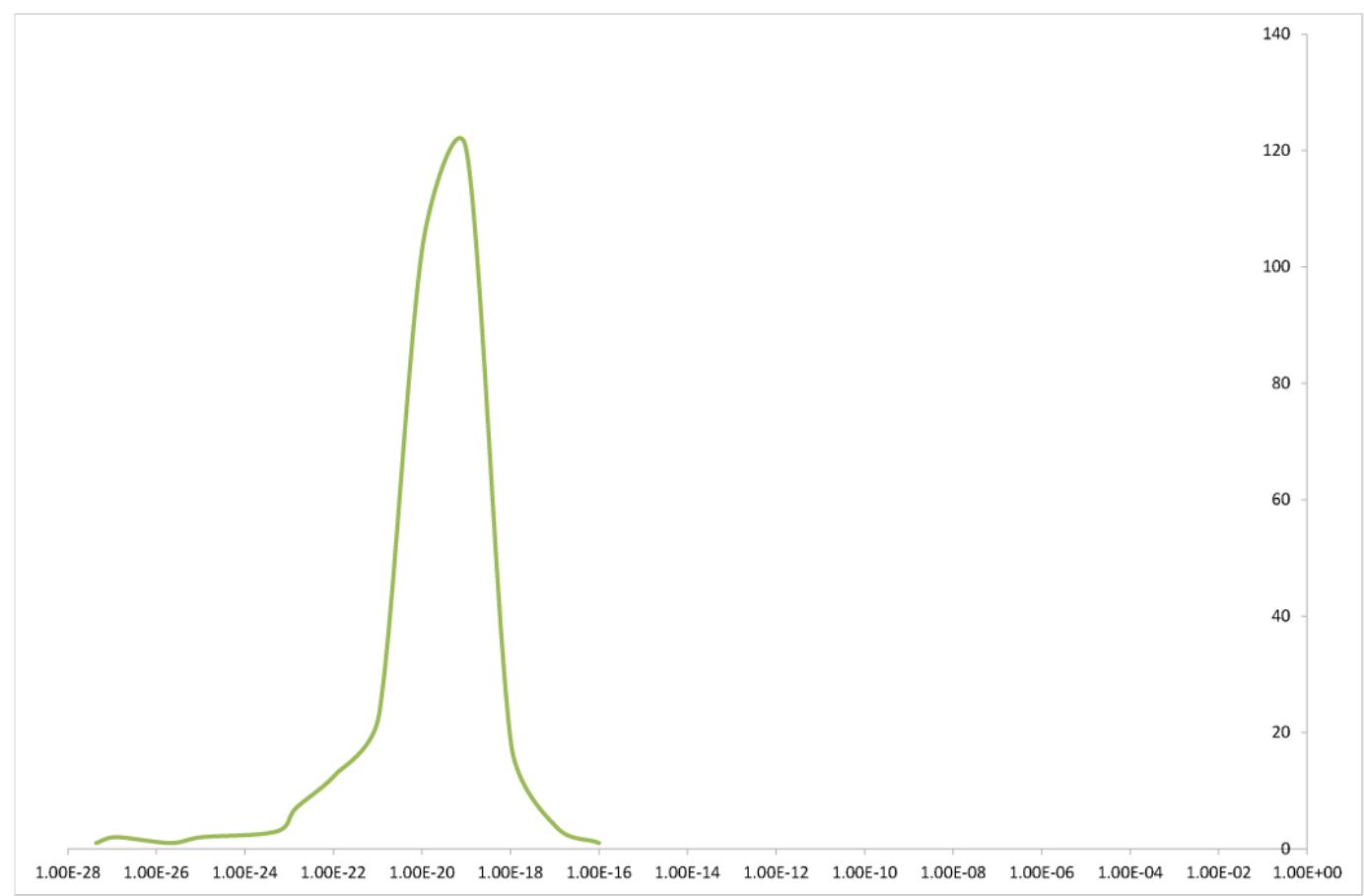

Figure 7. Chi Square curve of 300 results shows the confidence level of our data. 


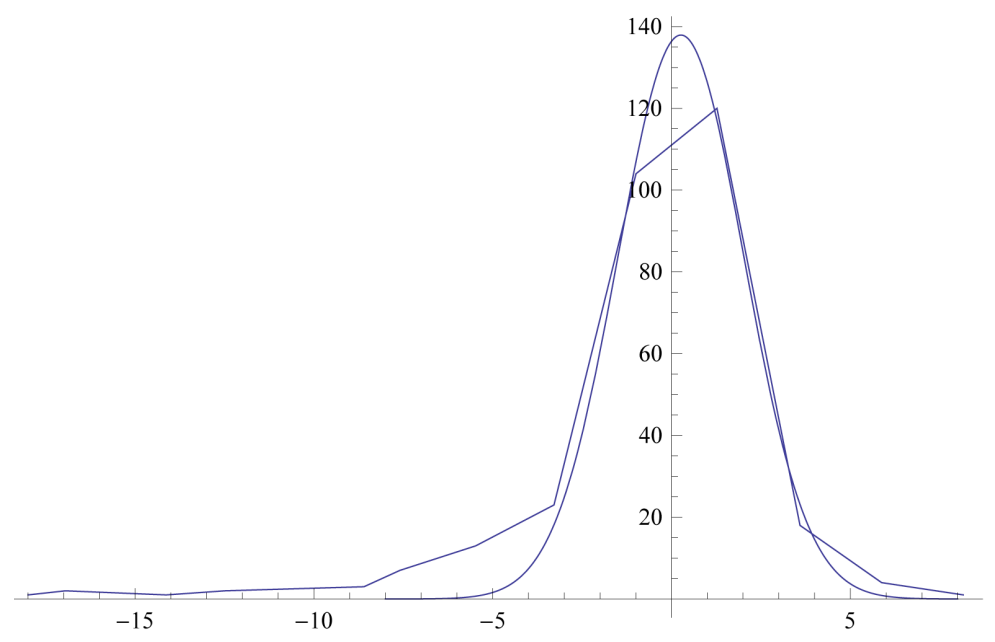

Figure 8. Fitness of Chi-square distribution with proposed data of Blasius equation.

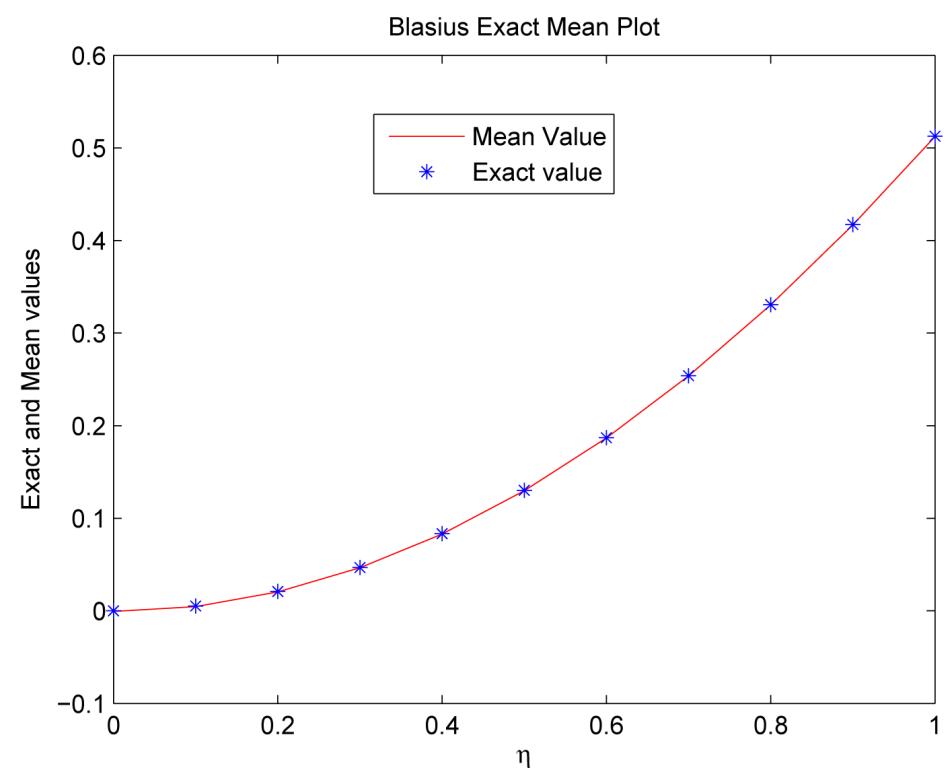

Figure 9. Mean VS Exact value of Blasius equation.

Thus it can be stated that proposed computing approach is reliable, effective and easily applicable for complex differential equation of order three. In our future work, we intend to use other hybrid computational intelligence algorithms like GA-SQP, GA-INP and GA-AST to solve these problems using Bessel's polynomial as active function.

\section{Acknowledgments}

The author would like to thanks Dr Sirj-ul-Islam for help in this research work.

\section{References}

[1] Howarth, L. (1938) On the Solution of the Laminar Boundary Layer Equations. Proceedings of the London Mathematical Society, 164, 547-579. http://dx.doi.org/10.1098/rspa.1938.0037

[2] Liao, S.J. (1999) An Explicit, Totally Analytic Approximate Solution for Blasius Viscous Flow Problems. International Journal of Non-Linear Mechanics, 34, 759-778. http://dx.doi.org/10.1016/S0020-7462(98)00056-0 
[3] Liao, S.J. (1992) The Proposed Homotopy Analysis Technique for the Solution of Nonlinear Problems. Ph.D. Thesis, Shanghai Jiao Tong University, Shanghai.

[4] Shishkin, G.I. (2001) Grid Approximation of the Solution to the Blasius Equation and of its Derivatives. Computational Mathematics and Mathematical Physics, 41, 37-54.

[5] Yu, L.T. and Kuang, C.C. (1998) The Solution of the Blasius Equation by the Differential Transformation Method. Mathematical and Computer Modeling, 28, 101-111.

[6] Schlichting, H. (1979) Boundary Layer Theory. McGraw-Hill, New York, 127-144.

[7] Coppel, W.A. (1960) On a Differential Equation of Boundary Layer Theory. Philosophical Transactions of the Royal Society A, 253, 101-136.

[8] Allan, F.M. and Abu-Saris, R.M. (1999) On the Existence and Non-Uniqueness of Nonhomogeneous Blasius Problem. Proceedings of the Second Pal. International Conference, Gorden and Breach, Newark.

[9] Howarth, L. (1938) On the Solution of the Laminar Boundary Layer Equations. Proceedings of the London Mathematical Society, 164, 547-579. http://dx.doi.org/10.1098/rspa.1938.0037

[10] Liao, S.J. (1999) An Explicit, Totally Analytic Approximate Solution for Blasius Viscous Flow Problems. International Journal of Non-Linear Mechanics, 34, 759-778. http://dx.doi.org/10.1016/S0020-7462(98)00056-0

[11] Khan, J.A. and Zahoor Raja, M.A. (2013) Artificial Intelligence based Solver for Governing Model of Radioactivity Cooling, Self-Gravitating Clouds and Clusters of Galaxies. Research Journal of Applied Sciences, Engineering and Technology, 6, 450-456.

[12] Zahoor Raja, M.A., Khan, J.A. and Qureshi, I.M. (2010) A New Stochastic Approach for Solution of Riccati Differential Equation of Fractional Order. Annals of Mathematics and Artificial Intelligence, 60, 229-250. http://dx.doi.org/10.1007/s10472-010-9222-x

[13] Zahoor Raja, M.A. and Samar, R. (2014) Numerical Treatment for Nonlinear MHD Jeffery-Hamel Problem Using Neural Networks Optimized with Interior Point Algorithm. Neurocomputing, 124, 178-193.

http://dx.doi.org/10.1016/j.neucom.2013.07.013 